\title{
Characterization of the Zonal Wind Flow in the Upper Atmosphere of Titan with the VLT
}

\author{
Régis Courtin ${ }^{1}$, David Luz ${ }^{1}$, Daniel Gautier ${ }^{1}$, Thierry Appourchaux ${ }^{2}$, \\ Jean-Pierre Lebreton ${ }^{2}$, Francesca Ferri ${ }^{3}$, Luisa Lara ${ }^{4}$ \\ Frédéric Hourdin ${ }^{5}$, Andreas Kaufer ${ }^{6}$ \\ ${ }^{1}$ LESIA-CNRS, Observatoire de Paris, 92195 Meudon Pal Cedex, France \\ ${ }^{2}$ ESTEC-European Space Agency, Noordwijk, The Netherlands \\ ${ }^{3}$ Universita di Padova, Padova, Italy \\ ${ }^{4}$ Instituto de Astrofisica de Andalucia, Grenada, Spain \\ ${ }^{5}$ Laboratoire de Météorologie Dynamique, Paris, France \\ ${ }^{6}$ European Southen Observatory, Santagio, Chile
}

\begin{abstract}
We report on recent efforts to characterize the zonal wind flow in the upper atmosphere of Titan from high resolution spectroscopic observations with the Very Large Telescope.
\end{abstract}

To characterize the zonal circulation of Titan's upper atmosphere, we have obtained high-resolution reflection spectra between 420 and $620 \mathrm{~nm}$ using the UVES echelle spectrometer at the focus of the VLT-UT2. The purpose of these observations was to detect the differential Doppler shift induced by the zonal wind flow in the back-scattered solar radiation from the Eastern and Western limbs. The measured spectra were analyzed with a velocity retrieval scheme developed for stellar accelerometry (Connes 1985), taking advantage of the large number of solar lines present in this spectral range. Similar observations were also made on Io to validate the velocity retrieval. A detailed analysis of the Io echellograms yields an equatorial velocity of $78.2 \pm 7.0 \mathrm{~m}-\mathrm{s}^{-1}$ (Civeit 2003), to be compared with the reference value of $74.7 \mathrm{~m}-\mathrm{s}^{-1}$. For Titan, a preliminary analysis shows the clear signature of a prograde circulation (i.e. in the direction of the solid body rotation) just south of the equator, with a wind velocity on the order of $80-100 \mathrm{~m}-\mathrm{s}^{-1}$, consistent with current model predictions (Hourdin et al. 1995). Radiative transfer calculations indicate that these measurements refer to altitudes between 50 and $250 \mathrm{~km}$. Another intriguing result, however, is that not all of our observations show the signature of super-rotation, which suggests that some process - yet to be identified - may be interfering with our ability to observe the wind flow at all times.

\section{References}

Civeit, T. 2003, Rapport de stage DESS, Obs. de Paris/Univ. Paris-Sud.

Connes, P. 1985, Ap\&SS, 110, 211

Hourdin, F., Talagrand, O., Sadourny, R. Courtin, R., Gautier, D., \& McKay, C. P. 1995 , Icarus, 117,358 